\title{
EXPRESSIVITY OF A COMMON FRAGILE SITE, fra(3)(p14.2), IN PATIENTS WITH CANCER AND OTHER DISEASES
}

\author{
Motoi Murata, ${ }^{1}$ Mikako Otsuka, ${ }^{1}$ Yukiko Hayakawa, ${ }^{1}$ \\ Ei-ichi TAKaHASH,${ }^{2}$ Hideo TsuJ,${ }^{2}$ and Tada-aki HoRI ${ }^{2}$ \\ ${ }^{1}$ Division of Epidemiology, Chiba Cancer Center Research Institute, \\ Chuo-ku, Chiba 260, Japan \\ ${ }^{2}$ Division of Genetics, National Institute of Radiological Sciences, \\ Anagawa, Inage-ku, Chiba 263, Japan
}

\begin{abstract}
Summary A population survey of a common folate-sensitive fragile site, fra(3)(p14.2), has been carried out on PHA-stimulated peripheral lymphocytes of patients with cancer and other diseases, under both culture conditions of folate deprivation and aphidicolin treatment. Overall findings regarding variability of expressivity due to age and sex were very similar to those obtained in a healthy population. The expression of fra(3)(p14.2) by folate deficient condition appeared hardly influenced by such exogenous factors as tobacco smoking habit, past histories of radiotherapy and chemotherapy, while it was associated with the unfavorable prognosis of cancers. Furthermore, proportion of those with higher expression was slightly but significantly larger in both lung and breast cancer patients. These findings suggest that some factors relevant to the expression of fra(3)(p14.2) may be associated with development and progression of certain kinds of cancer.
\end{abstract}

Key Words fra(3)(p14.2), folate deficiency, cancer patient, prognosis

\section{INTRODUCTION}

Among all common fragile sites on human chromosomes, the one on the short arm of chromosome 3, fra(3)(p14.2), or FRA3B in locus name, is most frequently expressed in such culture conditions as folate deficiency and aphidicolin (APC) treatment. In the previous paper, we reported on inter-individual variations of its expressivity and their underlying mechanisms, by using lymphocytes from more than one thousand healthy donors (Murata et al., 1990). The present study con-

Received Aprit 27, 1992; Accepted June 23, 1992. 
ducted a similar survey on patients of various diseases. Whereas there have been a few papers which showed a positive correlation with some cancers (Tedeschi et al., 1987; Furuya et al., 1989), the common fragile sites are not generally considered as having any biological significance.

Recently, on the other hand, a number of chromosome sites are clarified as a region of partial chromosome loss observed in cancer cells (Sager, 1989). Some of them are localized very close to the common fragile sites on the human chromosome map. For instances, loss of heterozygosity in breast cancer was reported at 1p32, 3p14, 11p14, 16q22 (Sager, 1989; Sato et al., 1991). These are all chromosome sites identical with the common fragile site. Especially, at 3p14, chromosomal deletion is observed in several kinds of cancer, i.e. cancers of the lung, breast, kidney, ovary and uterine cervix. Accordingly, fra(3)(p14.2) may possibly be a predisposing factor for a chromosomal change in cancer cells. We attempted to examine if any group of a specific disease would show a higher expressivity of this fragile site.

\section{MATERIALS AND METHODS}

The study subjects were a population of patients in Chiba Cancer Center Hospital. They were composed of the following two groups. One was a group of inpatients who were admitted and received a surgical treatment of cancer of various organs or non-malignant lesions mostly of gynecologic organs, from February, 1987 to the end of 1989. For this group, arterial blood samples, collected for the routine laboratory test prior to operation of patients, were utilized for the fragile site examination. The common fragile site was tested only under the folate deficient condition. The other was a group of outpatients who visited the Division of Respiratory Diseases from June, 1988 to May, 1991. They were diagnosed either of lung cancer or of other non-malignant respiratory diseases. For this group, the common fragile site was tested with both cultures of folate deprivation and APC treatment (Glover et al., 1984).

Including both groups, the folate deficient culture was performed for $753 \mathrm{pa}$ tients ( 351 males and 402 females) and the APC treated culture for 233 patients (147 males and 86 females). Their age was distributed in an older age range than the previously studied healthy population, with a mean of 52 years. For patients' epidemiologic and clinical characteristics, hospital records were referred to, which include family history of cancer, tobacco smoking habit, diagnosis, histories of radio- and chemotherapy, and prognosis.

For examining the expression of fragile site, $72 \mathrm{hr}$ whole blood culture was conducted as follows. In each culture, $0.5 \mathrm{ml}$ of heparinized whole blood sample was added in $4.5 \mathrm{ml}$ of culture medium and incubated in a $\mathrm{CO}_{2}(5 \%)$ incubator at $37^{\circ} \mathrm{C}$. The culture condition for folate deprivation was set up by using folic acid and thymidine-free Ham's F10 medium (M-F10, Gibco) containing 5\% fetal 
calf serum (FCS, Difco) and 2\% phytohemagglutinin (PHA, Wellcome). According to this assay system, folic acid deprivation in culture medium was rather incomplete, the final concentration being around $0.8 \mathrm{ng} / \mathrm{ml}$ which was about one fifth of that in blood serum. On the other hand, APC induced expression was examined by culturing lymphocytes in RPMI 1640 medium (Nissui) containing $20 \%$ FCS and 2\% PHA, and $0.1 \mu \mathrm{M}$ Aphidicolin (Wako) was added for the last $24 \mathrm{hr}$. To examine the background level of expression, the control culture without APC was also made. After $72 \mathrm{hr}$, chromosome preparations were made by a standard method following colchicine treatment $(2 \mathrm{hr})$, hypotonic treatment $(0.075 \mathrm{M} \mathrm{KCl})$, ethanol-acetic acid $(3: 1)$ fixation and air-drying. Fifty metaphase plates were scored for each culture condition on each subject and expression of fra(3)(p14.2) was counted. Expression frequency will be given by its number in 50 cells.

As in the previous study (Murata et al., 1990), the length of cell cycle was also tested for a sample of 50 patients. For this test, bromodeoxyuridine (BrdU, 7 $\mu \mathrm{g} / \mathrm{ml}$, Sigma) was added at the start of the RPMI 1640 culture without any other treatment. Then the chromosome slide was stained for $30 \mathrm{~min}$ in phosphate-buffer solution of Hoechst $33258(5 \mu \mathrm{g} / \mathrm{ml}, \mathrm{pH} 6.8)$, washed in the phosphate-buffer covered with a cover-glass and exposed to $360 \mathrm{~nm}$ light for $30 \mathrm{~min}$ on a hot-plate of $60^{\circ} \mathrm{C}$. After that, the slide was Giemsa-stained. With relative proportion of dark- and pale stained sister chromatids in each cell, it was determined whether the cell is the result of first (M1), second (M2) or third and more $(\mathrm{M} 3+$ ) cell division. For each subject, 200 cells were scored and the percentage of M3 + cells was calculated as an index of cell cycle.

\section{RESULTS}

The expression frequency of fra(3)(p14.2) under the folate deficient condition was varied among studied subjects in a Poisson-like distribution, as was observed in the healthy blood donors previously (Murata et al., 1990). But the frequency was, on the average, lower than that of the healthy subjects, being not accounted for by the difference of their age distributions. The differential expressivity between two populations was more remarkable in younger age classes (Fig. 1). Accordingly, the negative regression against the ages clearly demonstrated in the healthy population was rather obscured in the patients. On the other hand, the expression frequency of fra(3)(p14.2) with APC treatment appeared very comparable with that of the healthy population, if compared by age classes (Fig. 2). The significance of regression against patient's ages was marginal $(b=-0.032 \pm 0.018$, $\mathrm{p}=0.07$ ). Moreover, cell cycle also seems to be comparable between two populations, when compared by age classes, as is shown in Fig. 3.

Mean expression frequency in the folate deficient condition was compared between different groups classified by some characteristics of these patients (Table 1). Differences by such factors as a carrier state of heritable fragile sites, positive 
family history of cancer, tobacco smoking habit, past histories of radiotherapy and chemotherapy of cancers in recent 3 months, were all non-significant. There seems to be a difference among groups by the affected diseases, though not statisti-

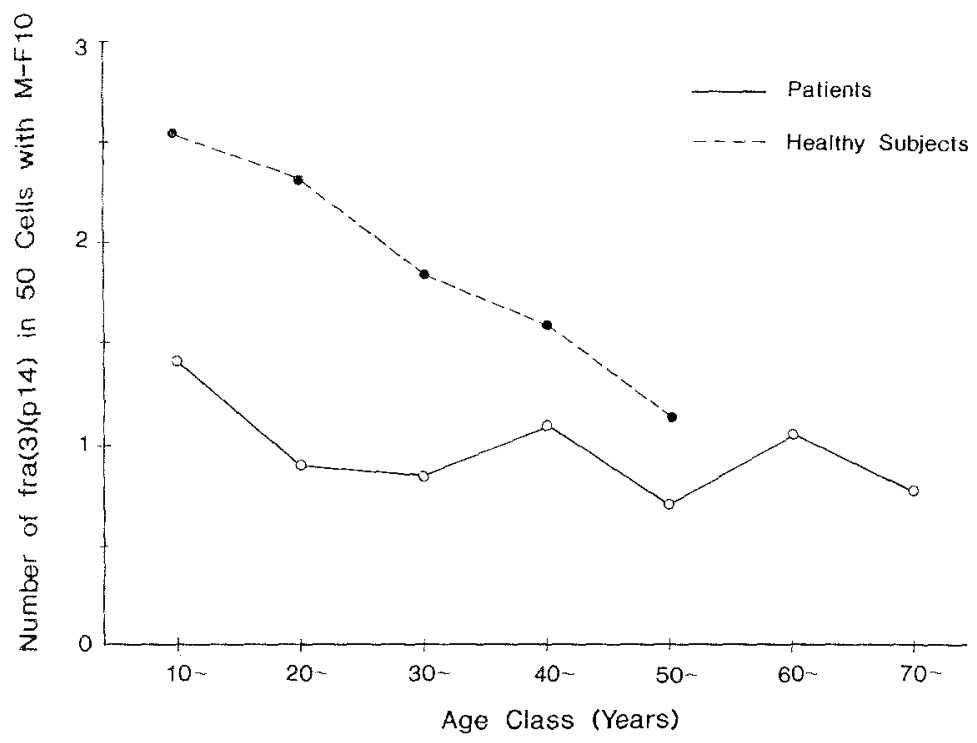

Fig. 1. Mean number of fra(3)(pi4.2) in 50 cells with folate deficient culture by age classes in patients and healthy subjects, with both sexes combined.

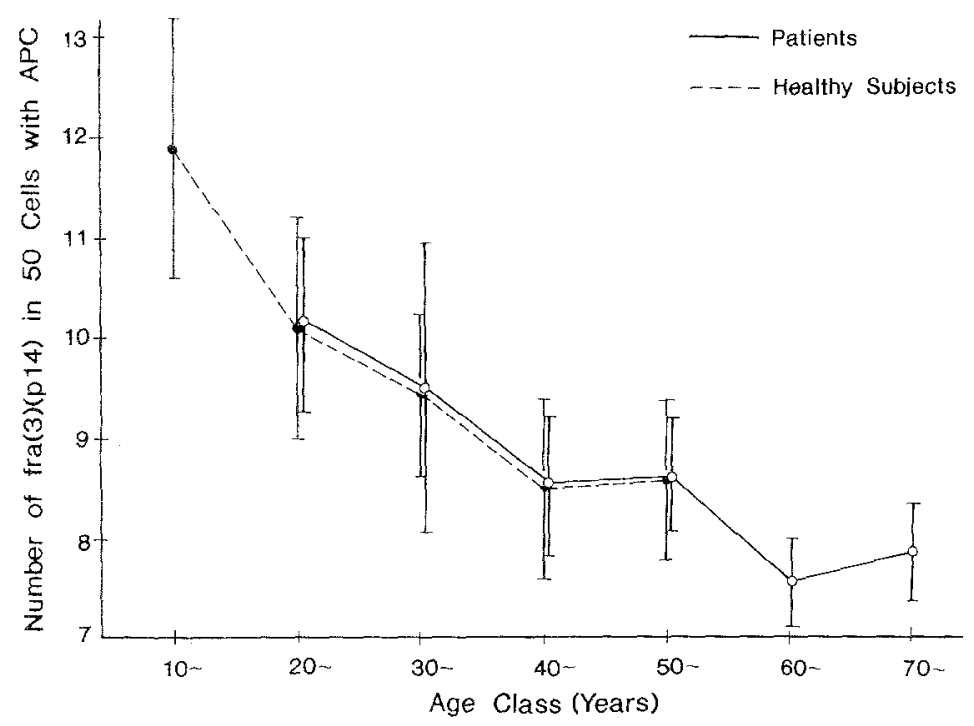

Fig. 2. Mean number of $\mathrm{fra}(3)(\mathrm{p} 14.2)$ in 50 cells with APC treatment by age classes in patients and healthy subjects, with both sexes combined. Vertical lines are a confidence interval of one standard error. 


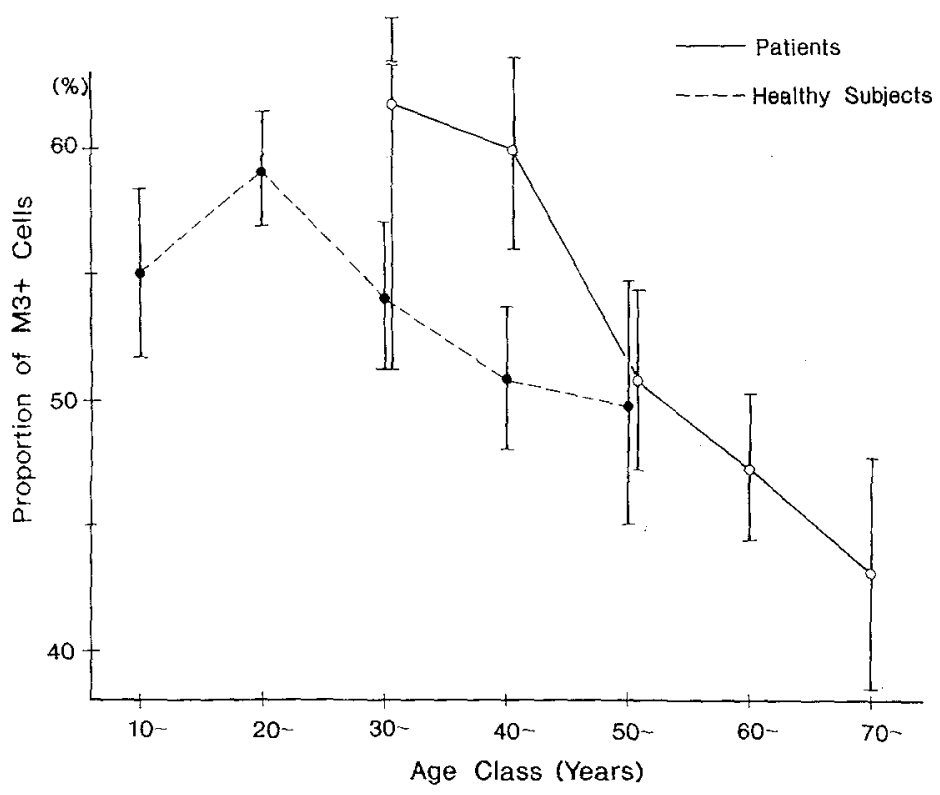

Fig. 3. Mean proportion of M3 + cells at $72 \mathrm{hr}$ of lymphocyte culture with RPMI 1640 medium by age classes, with both sexes combined. Vertical lines are a confidence interval of one standard error.

cally significant.

The only factor which showed a significant difference is the patient's prognosis. Those cancer patients who later deceased showed a higher mean expressivity than those who were not $(t=2.9$, d.f. $=183, p<0.01)$. This is clearly seen in Fig. 4 , where the cumulative survival rates of the cancer patients were calculated with the life table method (Cutler and Ederer, 1958) after having classified them by the fra(3)(p14.2) expressivity, i.e. $0-1,2-3$ and $4+$ in 50 cells. The curve for the group of highest expressivity is more rapidly declining than the rest till around two years after the chromosomal examination, their difference being significant at $5 \%$ level. After this period, however, no more excess mortality was seen in the former group. Thus, the expressivity seems to be correlated with the donor's mortality during a relatively short period.

So far the highest expressivity ( $44 \%$ ) among all studied subjects was presented by a patient of prostatic cancer with severe bone metastasis, whose age of 45 years was exceptionally young for this disease. Chromosome test on this particular patient was further repeated for seven times within the following three months, during which a high expressivity was retained, varying from 18 to $28 \%$. He was deceased after living 15 months from the time of first chromosome test.

Taking the factor of prognosis into account, only a group of inpatients were classified again by their affected diseases, because most of outpatients were not followed up in a complete manner. Table 2 shows frequency distribution of fra(3)- 
Table 1. Mean number of fra(3) (p14.2) in 50 metaphase cells by M-F10 culture in different classes of patients.

\begin{tabular}{|c|c|c|c|c|}
\hline & Traits & Classes & No. & Mean \\
\hline \multirow{2}{*}{\multicolumn{2}{|c|}{ Heritable fragile site }} & Carrier & 30 & 0.73 \\
\hline & & None & 670 & 0.95 \\
\hline \multirow{2}{*}{\multicolumn{2}{|c|}{ Family history of cancer }} & + & 143 & 0.82 \\
\hline & & - & 552 & 0.97 \\
\hline \multirow{2}{*}{\multicolumn{2}{|c|}{ Tobacco smoking (males) }} & + & 211 & 1.23 \\
\hline & & - & 102 & 0.89 \\
\hline \multirow{2}{*}{\multicolumn{2}{|c|}{ Radiotherapy history in 3 months }} & + & 39 & 1.05 \\
\hline & & - & 404 & 1.08 \\
\hline \multirow{2}{*}{\multicolumn{2}{|c|}{ Chemotherapy history in 3 months }} & + & 83 & 1.12 \\
\hline & & - & 360 & 1.07 \\
\hline \multirow[t]{8}{*}{ Disease } & Cancer of digestive organs & & 121 & 0.98 \\
\hline & Cancer of respiratory organs & & 126 & 1.19 \\
\hline & Cancer of bone, soft tissue, skin & & 29 & 1. 10 \\
\hline & Cancer of the breast & & 23 & 1.48 \\
\hline & Cancer of gynecologic organs & & 77 & 0.81 \\
\hline & Cancer of urogenital organs & & 32 & 1.66 \\
\hline & Other cancer & & 55 & 1.00 \\
\hline & Non-malignant diseases & & 290 & 0.75 \\
\hline \multirow{2}{*}{\multicolumn{2}{|c|}{$\begin{array}{ll}\text { Prognosis (cancer patients) } & \begin{array}{l}\text { survived } \\
\text { deceased }\end{array}\end{array}$}} & & 314 & $0.84 * *$ \\
\hline & & & 146 & 1.59 \\
\hline
\end{tabular}

** Difference between both values is significant at $1 \%$ level.

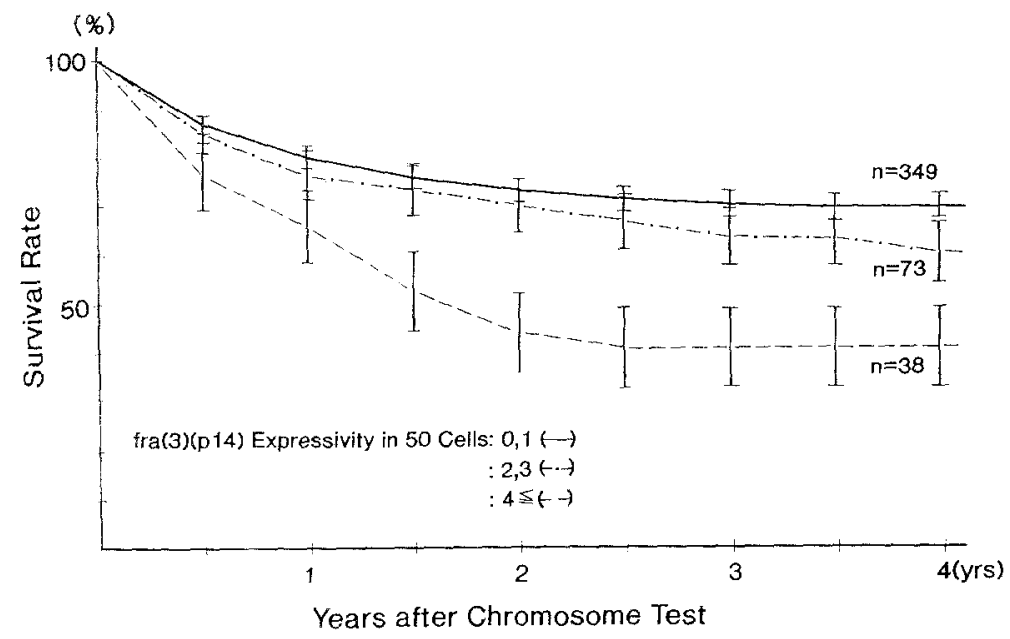

Fig. 4. Cumulative survival curves of cancer patients by their expressivity of fra(3)(p14.2) in lymphocyte culture with folate deprivation. Vertical lines are a confidence interval of one standard error. 
Table 2. Estimation of odds ratio (in parentheses) from cross tabulation of cases by disease groups and expression of fra(3)(p14.2) in comparison with a control group of patients with non-malignant diseases.

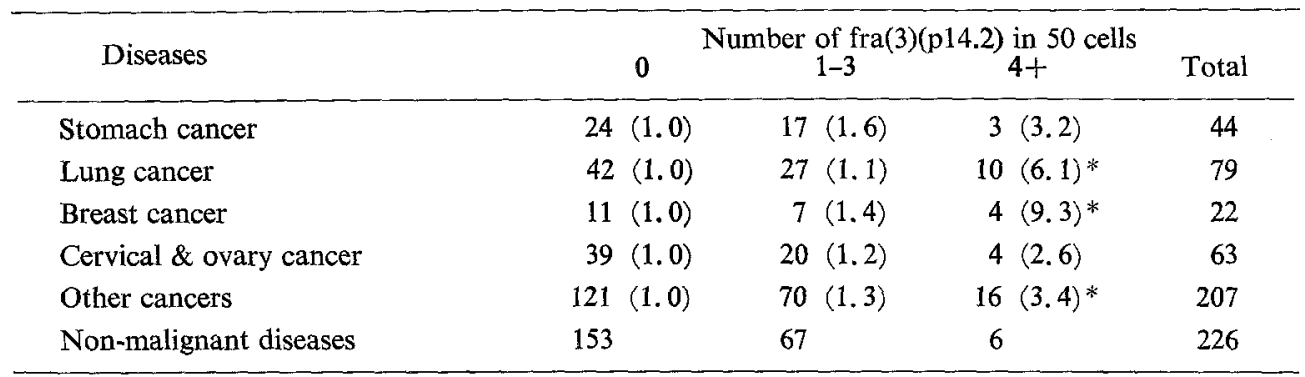

* Lower limit of $95 \%$ confidence interval of odds ratio exceeds 1.0 .

(p14.2) expression in 50 cells for different groups by the affected disease. Odds ratio was estimated for different cancer groups as compared with the group of nonmalignant diseases. For both lung cancer and breast cancer, the odds ratio in the class of 4 or more of fra(3)(p14.2) significantly exceeds 1.0. This statistical significance was barely retained if cases were confined to those who were alive. Whilst the group of other kinds of cancers also showed an odds ratio significantly larger than 1.0, it was not kept if limited to those cases who were alive.

Neither affected diseases (lung cancer vs. others) nor tobacco smoking habit did not influence the expressivity of fra(3)(p14.2) by the APC treatment (data not shown). Since almost all subjects of this test were out-patients, factors relevant to treatment of a disease and prognosis were not included in the study items. Thus we have no conclusive data regarding the influence of these factors on the expressivity of fra(3)(p14.2) by the APC treatment.

\section{DISCUSSION}

Exposure to such environmental factors as tobacco smoke, radiation and chemotherapeutic agents, all of which are already known to cause certain deleterious effects on chromosomes, had no effect on the expressivity a common fragile site. With respect to the tobacco smoking habit, the present result does not agree with the previously published one (Kao-Shan et al., 1987).

Evidence, both positive (Tedeschi et al., 1987; Furuya et al., 1989) and negative (Rao et al., 1988; Green et al., 1988; Porfirio et al., 1989; Kampmann et al., 1990), with respect to association of the expressivity of common fragile site with cancer has been reported with but a small number of subjects. In the present study, we tested the expression of fra(3)(p14.2) in a large number of unselected hospital patients, including cancers of the various organs. Thus, even though number of cases in each cancer was not so great, differences in the expression frequency can be tested statistically. 
First we noticed that the expressivity by folate deficiency was higher in cancer patients as a whole than those with other non-malignant diseases (Table 1). But this must be mainly owing to the factor of prognosis. Survival curves indicate that, for cancer patients, a higher expression of $\mathrm{fra}(3)(\mathrm{p} 14.2)$ in the folate deprivation is associated with death within a relatively short period after the chromosome test (Fig. 4). Those who later deceased were mostly patients with a severely metastasized, advanced cancer. Magnus (1967) has demonstrated, in a very large survey study, that the serum folic acid concentration in peripheral blood is, on the average, significantly reduced in the metastasized as compared with the non-metastasized cancer patients as well as with the controls. Since we already know that the folic acid concentration in blood is negatively correlated to the expression of fra(3)(p14.2) (Murata et al., 1990), the present result might be explained by possible folic acid deficiency in peripheral blood of the patients.

On the other hand, however, the result shown in Table 2 indicates that the expressivity of fra(3)(p14.2) was not so uniform among different cancer groups. Especially in lung and breast cancers, significantly higher expressivity was found in comparison with that of the non-malignant diseases. This significant difference was barely kept even after controlling for the factor of prognosis. Though we should be careful in interpreting the result because of small number of cases in each group, the higher expressivity may also be ascribed to other factors than a physiological condition resulted from an advanced state of the disease. This is rather interesting if connected with other findings that these cancers are very strongly associated with chromosome deletion at 3p14 in cancer cells (Sato et al., 1991; Yokoyama et al., 1992). Causal relation of fra(3)(p14.2) with this chromosomal change in cancer cells seems very likely.

For renal cell carcinoma, 3p14 has also been frequently reported as a site of partial chromosomal loss as well as a breakpoint of constitutional translocation $\mathrm{t}(3 ; 8)(\mathrm{p} 14.2 ; \mathrm{q} 24.1)$ in the familial case (Yamakawa et al., 1991). Glover et al. (1988) demonstrated that the expression of fra(3)(p14.2) with APC was altered in translocated chromosomes, indicating that the fragile site is very close to the breakpoint.

However, even though we found a significant elevation of expressivity in lung and breast cancer patients, number of cases with higher expressivity was not sufficiently large. In addition, Porfirio et al. (1989) found no difference of fra(3)(p14.2) expressivity with APC treatment between 35 lung cancer patients and 21 normal controls. Kampmann et al. (1990) also gave a negative result by using 26 patients of various cancers whose majority was lung and breast cancers. Thus the present result should be taken only as a suggestive evidence for positive association of a common fragile site with known chromosome abnormality in cancers.

Acknowledgments This work was supported in part by a Grant-in-Aid for Cancer Research from the Ministry of Education, Science and Culture, by that for the Comprehensive 10-Year Strategy for Cancer Control from the Ministry of Health and Welfare, and by the Smoking Re- 
search Foundation in Japan. We are very grateful to those members of the clinical branch of Chiba Cancer Center Hospital who helped us in providing blood samples.

\section{REFERENCES}

Cutler SJ, Ederer F (1958): Maximum utilization of the life table method in analyzing survival. J Chron Dis 8: 699-712

Furuya $T$, Ochi H, Watanabe $S$ (1989): Common fragile sites in chromosomes of bone marrow cells and peripheral blood lymphocytes from healthy persons and leukemia patients. Cancer Genet Cytogenet 43: 131-138

Glover TW, Berger C, Coyle J, Echo B (1984): DNA polymerase inhibition by aphidicolin induces gaps and breaks at common fragile sites in human chromosomes. Hum Genet 67: 136-142

Glover TW, Coyle-Morris JF, Li FP, Brown RS, Berger CS, Gemmill RM, Hecht F (1988): Translocation $t(3 ; 8)(\mathrm{p} 14.2 ; \mathrm{q} 24.1)$ in renal cell carcinoma affects expression of the common fragile site at 3p14 (FRA3B) in lymphocytes. Cancer Genet Cytogenet 31: 69-73

Green, RJ, Phillips DL, Chen ATL, Reidy JA, Ragab AH (1988): Effects of folate in culture medium on common fragile sites in lymphocyte chromosomes from normal and leukemic children. Hum Genet 81: 9-12

Kampmann T, Schmidt A, Rüdiger HW, Tan TL, Passarge E (1990): No difference in expression of chromosomal fragile sites in patients with solid malignant tumours and normal controls. Genes Chromos Cancer 2: 44-47

Kao-Shan C, Fine RL, Wang-Peng J, Lee EC, Chabner BA (1987): Increased fragile sites and sister chromatid exchanges in bone marrow and peripheral blood of young cigarette smokers. Cancer Res 47: 6278-6282

Magnus EM (1967): Folate activity in serum and red cell of patients with cancer. Cancer Res 27: 490-497

Murata M, Otsuka M, Hayakawa Y, Takahashi E, Tsuji H, Hori T (1990): A population cytogenetic study of a common fragile site, fra(3)(p14), in a healthy population. Jpn J Human Genet 35: 291-302

Porfirio B, Paladini P, Maccherini M, Gotti G, Cintorino M, De Marchi M (1989): Patients with different lung cancers show normal expression of fra(3)(p14) in aphidicolin-treated lymphocyte cultures. Cancer Genet Cytogenet 43: 95-101

Rao PN, Heerema NA, Palmer CG (1988): Expression of fragile sites in childhood acute lymphoblastic leukemia patients and normal controls. Hum Genet 79: 329-334

Sager R (1989): Tumor suppresser genes: The puzzle and the promise. Science 246: 1406-1412

Sato T, Akiyama, F, Sakamoto G, Kasumi F, Nakamura Y (1991): Accumulation of genetic alterations and progression of primary breast cancer. Cancer Res 51: 5794-5799

Tedeschi B, Porfirio B, Vernole P, Caporossi D, Dallpiccola B, Nicoletti B (1987): Common fragile sites: Their prevalence in subjects with constitutional and acquired chromosomal instability. Am J Med Genet 27: 471-482

Yamakawa K, Morita R, Takahashi E, Hori T, Ishikawa J, Nakamura Y (1991): A deletion mapping of the short arm of chromosome 3 in sporadic renal cell carcinoma. Cancer Res 51: 47074711

Yokoyama S, Yamakawa K, Tsuchiya E, Murata M, Sakiyama S, Nakamura Y (1992): Deletion mapping on the short arm of chromosome 3 in squamous cell carcinoma and adenocarcinoma of the lung. Cancer Res 52: 873-877 